\title{
含氮芥基查尔酮类衍生物的合成、晶体结构及抗肿瘤活性
}

\author{
方线文周丽珍成昭杨美盼杨秉勤* \\ (西北大学化学与材料科学学院 天然产物及功能分子教育部重点实验室 西安 710069)
}

\begin{abstract}
摘要 根据拼药原理, 将氮芥类抗肿瘤药物的药效基团 $N, N$-二(2-氯乙基)氨基拼接到查尔酮结构中, 合成出了一系列 新型含氮芥基的查尔酮衍生物 $3 \mathbf{a} \sim 3 \mathbf{r}$, 并用 ${ }^{1} \mathrm{H}$ NMR, IR, HRMS-ESI 及 X 射线对其结构进行了确证. 采用 MTT 法对所 合成的化合物进行了体外抗肿瘤活性测试，结果表明部分化合物对所选肿瘤细胞的增殖有较强的抑制作用，其中，化 合物 $3 \mathbf{j}$ 和 $3 \mathbf{l}$ 对 $\mathrm{K} 562$ 的 $\mathrm{IC}_{50}$ 值分别为 20.01 和 $18.6 \mu \mathrm{mol} / \mathrm{L}$; 对 $\mathrm{HepG} 2$ 的 $\mathrm{IC}_{50}$ 值分别为 43.46 和 $21.39 \mu \mathrm{mol} / \mathrm{L}$. 实验培 养并得到了目标产物 $3 \mathbf{e}$ 和 31 的晶体结构, $\mathrm{X}$ 射线分析表明化合物 $3 \mathbf{e}$ 和 31 都属三斜晶系, $P-1$ 空间群.
\end{abstract}

关键词 查尔酮; 氮芥; 合成; 晶体结构; 抗肿瘤活性

\section{Synthesis, Crystal Strutures and Antitumor Activity of Novel Ni- trogen Mustard-Linked Chalcones}

\author{
Fang, Xianwen Zhou, Lizhen Cheng, Zhao Yang, Meipan Yang, Bingqin* \\ (Key Laboratory of Synthetic and Natural Functional Molecule Chemistry of Ministry of Education, \\ Department of Chemistry and Materials Science, Northwest University, Xi'an 710069)
}

\begin{abstract}
A series of novel nitrogen mustard-linked chalcones $\mathbf{3 a} \sim 3 \mathbf{r}$ were synthesized by linking the $N, N$-bis(2- chloroethyl)amine pharmacophore of nitrogen mustards to the chalcone's framework. Their structures were characterized by ${ }^{1} \mathrm{H}$ NMR, IR, ESI-HRMS and X-ray techniques. The antitumor activities of the nitrogen mustard-linked chalcones were evaluated against K562 and HepG2 cell lines by an MTT assay. The results reveal that some of the title compounds exhibit potent anti-proliferative activities against selected tumor cells. Among which, compounds $\mathbf{3} \mathbf{j}$ against $\mathrm{K} 562$ and $\mathrm{HepG} 2$ with $\mathrm{IC}_{50}$ values of 20.01 and $43.46 \mu \mathrm{mol} / \mathrm{L}$, and $3 \mathrm{l}$ against $\mathrm{K} 562$ and HepG2 with $\mathrm{IC}_{50}$ values of 18.6 and $21.39 \mu \mathrm{mol} / \mathrm{L}$, respectively. Suitable crystals of $\mathbf{3 e}$ and $\mathbf{3 l}$ were obtained, and the X-ray diffraction analysis indicates that compounds $\mathbf{3 b}$ and $\mathbf{3 e}$ are both belong to triclinic, space group $P-1$.
\end{abstract}

Keywords chalcones; nitrogen mustard; synthesis; crystal structure; anti-tumor activity

查尔酮类衍生物是合成黄酮类等多种天然产物的 重要前体, 其化学结构为 1,3-二苯基丙烯酮, 以它为母 体的天然化合物广泛存在于甘草 ${ }^{[1]}$ 、红花、Licorice、啤 酒花等植物的根、叶和皮中. 查尔酮分子含 $\alpha, \beta$-烯酮结 构, 具有较大柔性, 能与不同的受体结合, 呈现出抗肿 瘤作用 ${ }^{[2]}$ 、抗炎作用 ${ }^{[3]}$ 、抗氧化作用 ${ }^{[4]}$ 、抗病毒作用 ${ }^{[5]}$ 、

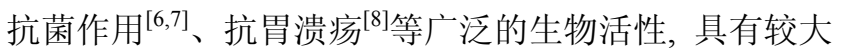
的临床应用潜力, 特别是其抗肿瘤活性及其作用机制更 是受到重视, 并开展了相关的研究 ${ }^{[9]}$. 研究表明, 查尔 酮类衍生物不仅可以抵抗多种致肿瘤物、促肿瘤物, 而 且可以抑制多种恶性肿瘤细胞的生长 ${ }^{[10 \sim 12]}$, 有望成为 低毒、高效的新型抗肿瘤药物. 但由于其水溶性和脂溶
性均较差，生物利用率也低，限制了其在临床上的应用. 因此有必要对查尔酮的结构进行化学修饰，以期得到活 性更高、毒性更小、结构新颖的新药.

氮芥类化合物是一类重要的烷基化抗肿瘤药物，在 临床上有广泛的应用 ${ }^{[13]}$. 该类药物的作用机制是通过 在体内形成高度活泼的氮丙环离子或碳正离子(亲电性 的强烷化剂), 进而与生物大分子(如 DNA, RNA 或某些 重要的酶类)中的富电子基团(如羟基、氨基、羧基、磷 酸基等)发生共价结合，使其丧失活性或使 DNA 分子发 生断裂，从而达到抗肿瘤目的 ${ }^{[13,14]}$. 目前已应用于临床 的氮芥类抗肿瘤药物主要有瘤可宁 ${ }^{[15]}$ 、美法仑 ${ }^{[16]}$ 、氮甲 [17]等. 氮芥类抗肿瘤药物结构主要由两个部分组成, 即

*E-mail: yangbq@nwu.edu.cn; fxw325@126.com

Received November 21, 2012; revised December 17, 2012; published online December 20, 2012

Project supported by the National Natural Science Foundation of China (No. 21172178).

国家自然科学基金(No.21172178)资助项目. 
烷基化部分和载体部分(Eq. 1). 根据载体的不同, 主要 可分为脂肪氮芥和芳香氮芥. 烷基化部分是抗肿瘤活性 的药效基团, 载体部分可以用以改善该类药物在体内的 吸收、分布等药动学性质, 从而影响药物的毒性、选择 性和抗肿瘤活性. 无论是芳香氮芥和脂肪氮芥都存在着 选择性差、毒性大的特点, 所以对氮芥的结构修饰尤其 是载体部分的修饰一直是药物化学的一个热点研究领 域 ${ }^{[18 \sim 21]}$.

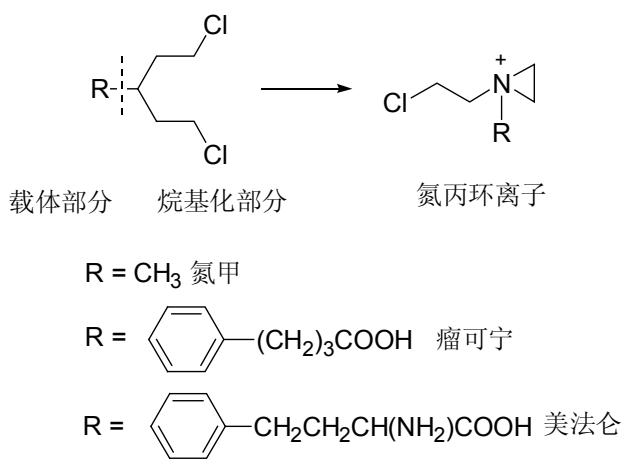

目前为止，将氮芥类抗肿瘤药物的烷基化部分(药 效基团)连接到查尔酮结构中未见文献报道. 本实验室 采用活性基团拼接法，将芳香氮芥药效基团导入查尔酮 载体结构上，合成出一系列新型的氮芥基连接的查尔酮 化合物. 合成路线如(Scheme 1), 所合成的目标化合物 均未见文献报道, 并对其进行了体外抗肿瘤活性研究.
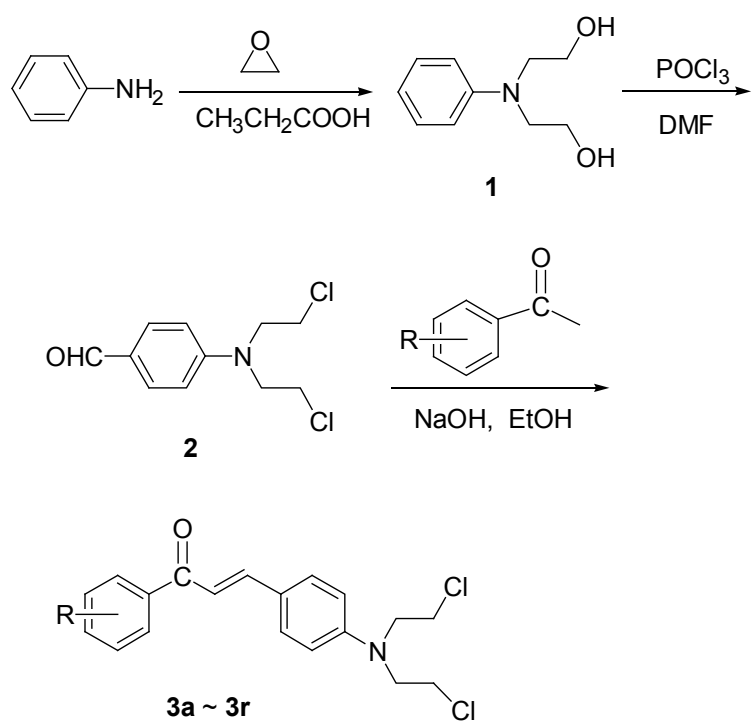

3a: $\mathrm{R}=3,4-\left(\mathrm{OCH}_{2} \mathrm{O}\right), 3 \mathbf{b}: \mathrm{R}=4-\mathrm{Cl}, 3 \mathrm{c}: \mathrm{R}=4-\mathrm{I}$, 3d: $\mathrm{R}=2-\mathrm{OH}, 3 \mathrm{e}: \mathrm{R}=4-\mathrm{Br}, 3 \mathrm{f}: \mathrm{R}=4-\mathrm{F}, 3 \mathrm{~g}: \mathrm{R}=4-\mathrm{CH}_{3}$, 3h: $\mathrm{R}=4-\mathrm{NO}_{2}, 3 \mathbf{i}: \mathrm{R}=4-\mathrm{OCH}_{2} \mathrm{CH}_{3}, 3 \mathbf{j}: \mathrm{R}=3,4-\left(\mathrm{OCH}_{3}\right)_{2}$, 3k: $\mathrm{R}=3,4-\left(\mathrm{CH}_{3}\right)_{2}, 3 \mathrm{I}: \mathrm{R}=4-\mathrm{OCH}_{3}, 3 \mathrm{~m}: \mathrm{R}=4-\mathrm{C}_{6} \mathrm{H}_{5}$, 3n: $\mathrm{R}=2-\mathrm{Br}-4-\mathrm{F}, 3 \mathrm{o}: \mathrm{R}=2,5-\left(\mathrm{CH}_{3}\right)_{2}, 3 \mathrm{p}: \mathrm{R}=\mathrm{H}$, 3q: $\mathrm{R}=2,4-\left(\mathrm{OCH}_{2} \mathrm{CH}=\mathrm{CH}_{2}\right)_{2}, 3 r: \mathrm{R}=4-\mathrm{N}\left(\mathrm{CH}_{2} \mathrm{CH}_{2} \mathrm{OH}\right)_{2}$

\section{1 实验部分}

\section{1 仪器与试剂}

X-4 型数字显微熔点测定仪(北京泰克仪器有限公 司，未经校正); INOVA-400 NMR 型核磁共振仪(TMS 内标, $\mathrm{CDCl}_{3}$ 或 DMSO- $d_{6}$ 为溶剂); EQUINOX-55 FTIR 型 红外光谱仪 ( $\mathrm{KBr}$ 压片); AXIMA-CFR ${ }^{\mathrm{TM}}$ plus MALDITOF 质谱仪; Bluker SMART APEXII-CCD X 射线单晶 衍射仪. 硅胶(200 300 目)为青岛海洋化工厂产品.

\section{2 合成}

\subsection{1中间体 $N, N$-二(2-羟乙基)-苯胺(1)的合成}

开启循环冷却泵, 使冷凝管温度降至 $-5{ }^{\circ} \mathrm{C}$, 二口 圆底烧瓶中加入苯胺 $18.6 \mathrm{~mL}(0.2 \mathrm{mmol})$, 水 $70 \mathrm{~mL}$ 和 丙酸 $5 \mathrm{~mL}$, 剧烈搅拌快速加入提前冷却的环氧乙烷 30 $\mathrm{mL}(0.59 \mathrm{mmol})$. 反应过夜, TLC 监测至原料点消失, 反 应液呈棕褐色. 将反应液倒入分液漏斗中，静置分层， 有机相分别用饱和 $\mathrm{Na}_{2} \mathrm{CO}_{3}$ 及水洗, 无水 $\mathrm{MgSO}_{4}$ 干燥过 夜. 减压蒸出溶剂, 残余物经硅胶柱色谱, 得白色固体. 收率 90\%, m.p. 55 57 ${ }^{\circ} \mathrm{C} ;{ }^{1} \mathrm{H}$ NMR $\left(400 \mathrm{MHz}, \mathrm{CDCl}_{3}\right)$ $\delta: 7.20(\mathrm{t}, J=6.8 \mathrm{~Hz}, 2 \mathrm{H}, \mathrm{ArH}), 6.71(\mathrm{t}, J=6.8 \mathrm{~Hz}, 1 \mathrm{H}$, $\operatorname{ArH}), 6.63(\mathrm{~d}, J=7.7 \mathrm{~Hz}, 2 \mathrm{H}, 2 \mathrm{H}), 4.54$ (s, 2H, OH), 3.73 (s, 4H, $\left.\mathrm{CH}_{2} \mathrm{CH}_{2} \mathrm{OH}\right), 3.48$ (s, 4H, $\left.\mathrm{CH}_{2} \mathrm{CH}_{2} \mathrm{OH}\right)$; IR (KBr) v: 3332, 2928, 2879, 1597, 1504, 1437, 1388, 1356, 1215, 1184, 1061, 1026, 752, $691 \mathrm{~cm}^{-1}$; HRMS (ESI) calcd for $\mathrm{C}_{10} \mathrm{H}_{16} \mathrm{NO}_{2}[\mathrm{M}+\mathrm{H}]^{+}$182.1176, found 182.1176 .

1.2.2 中间体 $N, N-$ 二(2-氯乙基)-4-氨基-苯甲醛(2)的 合成

装有干燥管的 $250 \mathrm{~mL}$ 圆底烧瓶中, 加入无水处理 的 DMF $30 \mathrm{~g}(410 \mathrm{mmol})$ 后, 搅拌下冰水浴中冷却 10 min. 撤去干燥管, 滴液漏斗缓慢滴加 $\mathrm{POCl}_{3} 16.6 \mathrm{~mL}$ (182.08 mmol)后, 冰浴中继续反应 $45 \mathrm{~min}$. 保持反应温 度为 $0{ }^{\circ} \mathrm{C}$, 一次性加入 $N, N$-二(2-羟乙基)-苯胺 $10 \mathrm{~g}$ $(55.18 \mathrm{mmol})$ 的 DMF $10 \mathrm{~g}(55.18 \mathrm{mmol})$ 溶液, 然后油浴 中慢慢升温至 $110{ }^{\circ} \mathrm{C}$ 反应 $3 \mathrm{~h}$, 冷去后倒入 $0.5 \mathrm{~L}$ 冰水 中并摚拌, 缓慢滴加 $1 \mathrm{~mol} \cdot \mathrm{L}^{-1} \mathrm{NaOH}$ 调 $\mathrm{pH}=7$, 有大量 沉淀产生. 抽滤, 滤饼用少量水洗, 粗品用乙醇重结晶, 得白色固体, 产率 $95 \%$. m.p. 81 83 ${ }^{\circ} \mathrm{C} ;{ }^{1} \mathrm{H}$ NMR (400 $\left.\mathrm{MHz}, \mathrm{CDCl}_{3}\right) \delta: 9.78(\mathrm{~s}, 1 \mathrm{H}, \mathrm{CHO}), 7.78(\mathrm{~d}, J=8.9 \mathrm{~Hz}$, $2 \mathrm{H}, \operatorname{ArH}), 6.75$ (d, $J=8.9 \mathrm{~Hz}, 2 \mathrm{H}, \operatorname{ArH}), 3.85$ (t, $J=6.9$ $\mathrm{Hz}, 4 \mathrm{H}, \mathrm{CH}_{2} \mathrm{CH}_{2} \mathrm{Cl}$ ), 3.69 (t, $J=6.9 \mathrm{~Hz}, 4 \mathrm{H}, \mathrm{CH}_{2} \mathrm{CH}_{2} \mathrm{Cl}$ ); IR (KBr) v: 3379, 2962, 1668, 1592, 1519, 1398, 1358, 1283, 1164, $813 \mathrm{~cm}^{-1}$; HRMS (ESI) calcd for $\mathrm{C}_{11} \mathrm{H}_{13}$ $\mathrm{Cl}_{2} \mathrm{NONa}[\mathrm{M}+\mathrm{Na}]^{+} 268.0268$, found 268.0280.

Scheme 1 
1.2.3 目标物含氮芥基查尔酮类衍生物 $\mathbf{3 a} \sim \mathbf{3 r}$ 的合 成

$25 \mathrm{~mL}$ 单口圆底烧瓶中加入取代苯乙酮 $(1 \mathrm{mmol})$ 和 乙醇 $3 \mathrm{~mL}$, 冰浴下搅拌 $20 \mathrm{~min}$ 后, 加入 $N, N$-二(2-氯乙 基)-4-氨基-苯甲醛(2) $0.25 \mathrm{~g}$ (1 mmol), 搅拌使之溶解. 缓慢滴加 $1 \mathrm{~mL} 10 \% \mathrm{NaOH}$ 溶液(约 $10 \mathrm{~min}$ 滴完). TLC 监 测, 至原料点消失, 反应液用乙酸乙酯萃取, 无水 $\mathrm{MgSO}_{4}$ 干燥, 减压蒸出溶剂, 残余物经硅胶柱色谱, 得 目标产物.

(E)-4-[N,N-二(2-氯乙基)-4-氨基-苯基]-1-[3',4'-(亚 甲二氧基)苯基]-2-丙烯-1-酮(3a): 黄色固体, 收率 $84 \%$. m.p. 91 93 ${ }^{\circ} \mathrm{C} ;{ }^{1} \mathrm{H}$ NMR (400 MHz, $\left.\mathrm{CDCl}_{3}\right) \delta: 7.75(\mathrm{~d}$, $J=15.4 \mathrm{~Hz}, 1 \mathrm{H}, \mathrm{COCH}=\mathrm{CH}), 7.64(\mathrm{~d}, J=8.0 \mathrm{~Hz}, 1 \mathrm{H}$, $\mathrm{COCH}=\mathrm{CH}), 7.60 \sim 7.48(\mathrm{~m}, 3 \mathrm{H}, \mathrm{ArH}), 7.45 \sim 7.29(\mathrm{~m}$, $1 \mathrm{H}, \mathrm{ArH}), 6.89$ (d, $J=8.1 \mathrm{~Hz}, 1 \mathrm{H}, \mathrm{ArH}), 6.69$ (d, $J=8.5$ $\mathrm{Hz}, 2 \mathrm{H}, \mathrm{ArH}), 6.05$ (d, $\left.J=5.6 \mathrm{~Hz}, 2 \mathrm{H}, \mathrm{OCH}_{2} \mathrm{O}\right), 3.81(\mathrm{dd}$, $\left.J=14.9,8.0 \mathrm{~Hz}, 4 \mathrm{H}, \mathrm{CH}_{2} \mathrm{CH}_{2} \mathrm{Cl}\right), 3.66$ (t, $J=6.6 \mathrm{~Hz}, 4 \mathrm{H}$, $\mathrm{CH}_{2} \mathrm{CH}_{2} \mathrm{Cl}$ ); IR (KBr) v: 3068, 2921, 1649, 1574, 1513, 1437, 1394, 1352, 1243, 1171, 1105, 1031, 926, 804, 741 $\mathrm{cm}^{-1}$; HRMS (ESI) calcd for $\mathrm{C}_{20} \mathrm{H}_{19} \mathrm{Cl}_{2} \mathrm{NO}_{3} \mathrm{Na}[\mathrm{M}+\mathrm{Na}]^{+}$ 414.0634, found 414.0651.

(E)-4-[N,N-二(2-氯乙基)-4-氨基-苯基]-1-(4'-氯苯 基)-2-丙烯-1-酮(3b): 黄色固体, 收率 87\%. m.p. 156 $157{ }^{\circ} \mathrm{C} ;{ }^{1} \mathrm{H}$ NMR (400 MHz, $\mathrm{CDCl}_{3}$ ) $\delta: 7.95$ (d, $J=8.3$ $\mathrm{Hz}, 2 \mathrm{H}, \mathrm{ArH}), 7.77$ (d, J=15.5 Hz, 1H, $\mathrm{COCH}=\mathrm{CH})$, 7.57 (d, $J=8.6 \mathrm{~Hz}, 2 \mathrm{H}, \operatorname{ArH}), 7.47$ (d, $J=8.2 \mathrm{~Hz}, 2 \mathrm{H}$, $\operatorname{ArH}), 7.32(\mathrm{~d}, J=15.5 \mathrm{~Hz}, 1 \mathrm{H}, \mathrm{COCH}=\mathrm{CH}), 6.70(\mathrm{~d}, J=$ $8.7 \mathrm{~Hz}, 2 \mathrm{H}, \mathrm{ArH}), 3.81$ (t, $J=6.9 \mathrm{~Hz}, 4 \mathrm{H}, \mathrm{CH}_{2} \mathrm{CH}_{2} \mathrm{Cl}$ ), $3.67\left(\mathrm{t}, J=6.9 \mathrm{~Hz}, 4 \mathrm{H}, \mathrm{CH}_{2} \mathrm{CH}_{2} \mathrm{Cl}\right)$; IR ( $\left.\mathrm{KBr}\right) v: 3445$, 2960, 1647, 1582, 1557, 1517, 1393, 1341, 1252, 1177, 1085, 980, 817, $807 \mathrm{~cm}^{-1}$; HRMS (ESI) calcd for $\mathrm{C}_{19} \mathrm{H}_{18} \mathrm{Cl}_{3} \mathrm{NONa}[\mathrm{M}+\mathrm{Na}]^{+}$404.0346, found 404.0336.

(E)-4-[N, $N$-二(2-氯乙基)-4-氨基-苯基]-1-(4'-碘苯 基)-2-丙烯-1-酮(3c)：黄色固体，收率 82\%. m.p. 93 94 ${ }^{\circ} \mathrm{C}$; ${ }^{1} \mathrm{H}$ NMR $\left(400 \mathrm{MHz}, \mathrm{CDCl}_{3}\right) \delta: 7.85(\mathrm{~d}, J=7.9 \mathrm{~Hz}, 2 \mathrm{H}$, $\mathrm{ArH}), 7.78(\mathrm{~d}, J=7.2 \mathrm{~Hz}, 1 \mathrm{H}, \mathrm{COCH}=\mathrm{CH}), 7.75 \sim 7.70$ (m, 2H, ArH), 7.57 (d, J=8.4 Hz, 2H, ArH), 7.31 (s, 1H, $\mathrm{COCH}=\mathrm{CH}), 6.73(\mathrm{dd}, J=14.2,8.6 \mathrm{~Hz}, 2 \mathrm{H}, \mathrm{ArH}), 3.83$ $\left(\mathrm{dt}, J=13.8,6.8 \mathrm{~Hz}, 4 \mathrm{H}, \mathrm{CH}_{2} \mathrm{CH}_{2} \mathrm{Cl}\right), 3.67(\mathrm{t}, J=6.2 \mathrm{~Hz}$, $4 \mathrm{H}, \mathrm{CH}_{2} \mathrm{CH}_{2} \mathrm{Cl}$ ); IR (KBr) v: 3442, 2926, 1645, 1579, 1515, 1393, 1348, 1171, 1028, 994, $804 \mathrm{~cm}^{-1}$; HRMS (ESI) calcd for $\mathrm{C}_{19} \mathrm{H}_{18} \mathrm{Cl}_{2} \mathrm{INONa}[\mathrm{M}+\mathrm{Na}]^{+}$495.9702, found 495.9684 .

(E)-4-[ $N, N$-二(2-氯乙基)-4-氨基-苯基]-1-(2'-差基苯基)-2-丙烯-1-酮(3d): 橙红色固体, 收率 65\%. m.p.
105 $107{ }^{\circ} \mathrm{C} ;{ }^{1} \mathrm{H}$ NMR (400 MHz, $\left.\mathrm{CDCl}_{3}\right) \delta: 7.96 \sim 7.84$ $(\mathrm{m}, 2 \mathrm{H}, \mathrm{COCH}=\mathrm{CH}, \mathrm{ArH}), 7.59(\mathrm{~d}, J=8.7 \mathrm{~Hz}, 2 \mathrm{H}, \mathrm{ArH})$, $7.50(\mathrm{dd}, J=17.4,6.5 \mathrm{~Hz}, 2 \mathrm{H}, \operatorname{ArH}), 7.02$ (d, $J=8.3 \mathrm{~Hz}$, $1 \mathrm{H}, \mathrm{COCH}=\mathrm{CH}), 6.94(\mathrm{t}, J=7.6 \mathrm{~Hz}, 1 \mathrm{H}, \mathrm{ArH}), 6.71(\mathrm{~d}$, $J=8.7 \mathrm{~Hz}, 2 \mathrm{H}, \mathrm{ArH}), 3.81\left(\mathrm{t}, J=6.9 \mathrm{~Hz}, 4 \mathrm{H}, \mathrm{CH}_{2} \mathrm{CH}_{2} \mathrm{Cl}\right)$, $3.67\left(\mathrm{t}, J=6.9 \mathrm{~Hz}, 4 \mathrm{H}, \mathrm{CH}_{2} \mathrm{CH}_{2} \mathrm{Cl}\right), 3.48$ (s, $\left.1 \mathrm{H}, \mathrm{OH}\right)$; IR (KBr) v: 3446, 2962, 1629, 1548, 1514, 1483, 1336, 1202, $1175,1147,1022,812,759 \mathrm{~cm}^{-1}$; HRMS (ESI) calcd for $\mathrm{C}_{19} \mathrm{H}_{19} \mathrm{Cl}_{2} \mathrm{NO}_{2} \mathrm{Na}[\mathrm{M}+\mathrm{Na}]^{+}$386.0685, found 386.0696.

(E)-4-[N,N-二(2-氯乙基)-4-氨基-苯基]-1-(4'-溴苯 基)-2-丙烯-1-酮(3e)：黄色固体，收率 87\%. m.p. 149 $151{ }^{\circ} \mathrm{C} ;{ }^{1} \mathrm{H}$ NMR (400 MHz, $\left.\mathrm{CDCl}_{3}\right) \delta: 7.87$ (d, $J=8.4$ $\mathrm{Hz}, 2 \mathrm{H}, \mathrm{ArH}), 7.79 \sim 7.74(\mathrm{~m}, 1 \mathrm{H}, \mathrm{COCH}=\mathrm{CH}), 7.63(\mathrm{~d}$, $J=8.4 \mathrm{~Hz}, 2 \mathrm{H}, \mathrm{ArH}), 7.56(\mathrm{~d}, J=8.8 \mathrm{~Hz}, 2 \mathrm{H}, \mathrm{ArH}), 7.30$ $(\mathrm{d}, J=15.5 \mathrm{~Hz}, 1 \mathrm{H}, \mathrm{COCH}=\mathrm{CH}), 6.72(\mathrm{dd}, J=15.5,8.9$ $\mathrm{Hz}, 2 \mathrm{H}, \mathrm{ArH}$ ), 3.81 (dd, $J=13.7,6.8 \mathrm{~Hz}, 4 \mathrm{H}, \mathrm{CH}_{2} \mathrm{CH}_{2} \mathrm{Cl}$ ), $3.70 \sim 3.64\left(\mathrm{~m}, 4 \mathrm{H}, \mathrm{CH}_{2} \mathrm{CH}_{2} \mathrm{Cl}\right)$; IR (KBr) v: 3445, 2958, 2925, 1644, 1582, 1515, 1392, 1350, 1248, 1171, 1065, 994, 805, $742 \mathrm{~cm}^{-1}$; HRMS (ESI) calcd for $\mathrm{C}_{19} \mathrm{H}_{18} \mathrm{BrCl}_{2-}$ $\mathrm{NONa}[\mathrm{M}+\mathrm{Na}]^{+}$449.9818, found 449.9807 .

(E)-4-[ $N, N$-二(2-氯乙基)-4-氨基-苯基]-1-(4'-氟苯 基)-2-丙烯-1-酠(3f): 黄色片状固体，收率 84\%， m.p. 93 95 ${ }^{\circ} \mathrm{C} ;{ }^{1} \mathrm{H}$ NMR (400 MHz, $\left.\mathrm{CDCl}_{3}\right) \delta: 8.08 \sim 8.00$ $(\mathrm{m}, 2 \mathrm{H}, \mathrm{ArH}), 7.77(\mathrm{~d}, J=15.5 \mathrm{~Hz}, 1 \mathrm{H}, \mathrm{COCH}=\mathrm{CH})$, 7.56 (d, $J=8.8 \mathrm{~Hz}, 2 \mathrm{H}, \mathrm{ArH}), 7.33$ (d, $J=15.5 \mathrm{~Hz}, 1 \mathrm{H}$, $\mathrm{ArH}), 7.16$ (t, $J=8.6 \mathrm{~Hz}, 2 \mathrm{H}, \mathrm{ArH}, \mathrm{COCH}=\mathrm{CH}), 6.70(\mathrm{~d}$, $J=8.8 \mathrm{~Hz}, 2 \mathrm{H}, \mathrm{ArH}), 3.80$ (t, $\left.J=6.9 \mathrm{~Hz}, 4 \mathrm{H}, \mathrm{CH}_{2} \mathrm{CH}_{2} \mathrm{Cl}\right)$, 3.67 (t, $\left.J=6.9 \mathrm{~Hz}, 4 \mathrm{H}, \mathrm{CH}_{2} \mathrm{CH}_{2} \mathrm{Cl}\right)$; IR (KBr) v: 3418, 3070, 3023, 2924, 1645, 1593, 1566, 1512, 1342, 1210, $1177,1151,1025,984,812,738 \mathrm{~cm}^{-1}$; HRMS (ESI) calcd for $\mathrm{C}_{19} \mathrm{H}_{18} \mathrm{Cl}_{2} \mathrm{FNONa}[\mathrm{M}+\mathrm{Na}]^{+}$388.0642, found 388.0649 .

(E)-4-[ $N, N$-二(2-氯乙基)-4-氨基-苯基]-1-(4'-甲基苯 基)-2-丙烯-1-酥(3g): 黄色固体, 收率 91\%. m.p. 132 $133{ }^{\circ} \mathrm{C} ;{ }^{1} \mathrm{H}$ NMR (400 MHz, $\mathrm{CDCl}_{3}$ ) $\delta: 7.93$ (d, $J=8.1$ $\mathrm{Hz}, 2 \mathrm{H}, \mathrm{ArH}), 7.76(\mathrm{~d}, J=15.5 \mathrm{~Hz}, 1 \mathrm{H}, \mathrm{COCH}=\mathrm{CH})$, 7.57 (d, $J=8.8 \mathrm{~Hz}, 2 \mathrm{H}, \operatorname{ArH}), 7.37$ (d, $J=15.5 \mathrm{~Hz}, 1 \mathrm{H}$, $\mathrm{COCH}=\mathrm{CH}), 7.30(\mathrm{~d}, J=8.0 \mathrm{~Hz}, 2 \mathrm{H}, \mathrm{ArH}), 6.70(\mathrm{~d}, J=$ $8.8 \mathrm{~Hz}, 2 \mathrm{H}, \mathrm{ArH}), 3.80\left(\mathrm{t}, J=7.0 \mathrm{~Hz}, 4 \mathrm{H}, \mathrm{CH}_{2} \mathrm{CH}_{2} \mathrm{Cl}\right)$, 3.67 ( $\left.\mathrm{t}, J=6.9 \mathrm{~Hz}, 4 \mathrm{H}, \mathrm{CH}_{2} \mathrm{CH}_{2} \mathrm{Cl}\right), 2.43$ (s, 3H, $\mathrm{OCH}_{3}$ ); IR (KBr) v: 3026, 2958, 2923, 1645, 1605, 1577, 1517, 1434, 1344, 1252, 1177, 1032, 982, $806 \mathrm{~cm}^{-1}$; HRMS (ESI) calcd for $\mathrm{C}_{20} \mathrm{H}_{21} \mathrm{Cl}_{2} \mathrm{NONa}[\mathrm{M}+\mathrm{Na}]^{+}$384.0892, found 384.0886 .

(E)-4-[ $N, N$-二(2-氯乙基)-4-氨基-苯基]-1-(4'-硝基苯 
基)-2-丙烯-1-酮(3h)：红褐色固体，收率 81\%. m.p. 197 $199{ }^{\circ} \mathrm{C} ;{ }^{1} \mathrm{H}$ NMR (400 MHz, DMSO- $\left.d_{6}\right) \delta: 7.87$ (d, $J=8.4 \mathrm{~Hz}, 2 \mathrm{H}, \mathrm{ArH}), 7.77$ (d, $J=15.4 \mathrm{~Hz}, 1 \mathrm{H}, \mathrm{COCH}=$ $\mathrm{CH}), 7.63$ (d, $J=8.4 \mathrm{~Hz}, 2 \mathrm{H}, \mathrm{ArH}), 7.56$ (d, $J=8.8 \mathrm{~Hz}$, $2 \mathrm{H}, \mathrm{ArH}), 7.30(\mathrm{~d}, J=15.5 \mathrm{~Hz}, 1 \mathrm{H}, \mathrm{COCH}=\mathrm{CH}), 6.71(\mathrm{t}$, $J=7.7 \mathrm{~Hz}, 2 \mathrm{H}, \mathrm{ArH}), 3.86 \sim 3.78\left(\mathrm{~m}, 4 \mathrm{H}, \mathrm{CH}_{2} \mathrm{CH}_{2} \mathrm{Cl}\right)$, $3.71 \sim 3.64\left(\mathrm{~m}, 4 \mathrm{H}, \mathrm{CH}_{2} \mathrm{CH}_{2} \mathrm{Cl}\right)$; IR (KBr) v: 3448, 2924, 1647, 1597, 1568, 1516, 1340, 1253, 1206, 1033, 848, 817, $764 \mathrm{~cm}^{-1}$; HRMS (ESI) calcd for $\mathrm{C}_{19} \mathrm{H}_{18} \mathrm{Cl}_{2} \mathrm{~N}_{2} \mathrm{O}_{3} \mathrm{Na}[\mathrm{M}+$ $\mathrm{Na}]^{+}$415.0587, found 415.0570.

(E)-4-[ $N, N$-二(2-氯乙基)-4-氨基-苯基]-1-(4'-乙氧基 苯基)-2-丙烯-1-酮(3i)：黄色固体，收率 84\%. m.p. 74 $76{ }^{\circ} \mathrm{C} ;{ }^{1} \mathrm{H}$ NMR $\left(400 \mathrm{MHz}, \mathrm{CDCl}_{3}\right) \delta: 8.02$ (d, $J=8.5 \mathrm{~Hz}$, $1 \mathrm{H}, \mathrm{COCH}=\mathrm{CH}), 7.77(\mathrm{dd}, J=11.9,7.9 \mathrm{~Hz}, 2 \mathrm{H}, \mathrm{ArH})$, 7.57 (d, $J=8.5 \mathrm{~Hz}, 2 \mathrm{H}, \mathrm{ArH}), 7.39$ (d, $J=15.5 \mathrm{~Hz}, 1 \mathrm{H}$, $\mathrm{COCH}=\mathrm{CH}), 6.96(\mathrm{~d}, J=8.5 \mathrm{~Hz}, 2 \mathrm{H}, \mathrm{ArH}), 6.72(\mathrm{dd}, J=$ $14.4,8.7 \mathrm{~Hz}, 2 \mathrm{H}, \mathrm{ArH}), 4.12$ (d, $J=7.0 \mathrm{~Hz}, 2 \mathrm{H}, \mathrm{OCH}_{2}-$ $\left.\mathrm{CH}_{3}\right), 3.86 \sim 3.78\left(\mathrm{~m}, 4 \mathrm{H}, \mathrm{CH}_{2} \mathrm{CH}_{2} \mathrm{Cl}\right), 3.67$ (dd, $J=12.1$, $6.3 \mathrm{~Hz}, 4 \mathrm{H}, \mathrm{CH}_{2} \mathrm{CH}_{2} \mathrm{Cl}$ ), 1.45 (t, $J=6.9 \mathrm{~Hz}, 3 \mathrm{H}, \mathrm{OCH}_{2}-$ $\mathrm{CH}_{3}$ ); IR (KBr) v: 3444, 2966, 2926, 1646, 1595, 1515, 1465, 1396, 1343, 1256, 1223, 1161, 1030, 970, $809 \mathrm{~cm}^{-1}$; HRMS (ESI) calcd for $\mathrm{C}_{21} \mathrm{H}_{23} \mathrm{Cl}_{2} \mathrm{NO}_{2} \mathrm{Na}[\mathrm{M}+\mathrm{Na}]^{+}$ 414.0998, found 414.0993.

(E)-4-[ $N, N$-二(2-氯乙基)-4-氨基-苯基]-1-(3',4'-二甲 氧基苯基)-2-丙烯-1-酮(3j): 黄色固体, 收率 87\%. m.p. $129 \sim 131{ }^{\circ} \mathrm{C} ;{ }^{1} \mathrm{H}$ NMR $\left(400 \mathrm{MHz}, \mathrm{CDCl}_{3}\right) \delta: 7.77$ (d, $J=$ $15.5 \mathrm{~Hz}, 1 \mathrm{H}, \mathrm{COCH}=\mathrm{CH}), 7.67(\mathrm{dd}, J=8.3,1.7 \mathrm{~Hz}, 1 \mathrm{H}$, ArH), 7.62 (d, $J=1.6 \mathrm{~Hz}, 1 \mathrm{H}, \operatorname{ArH}), 7.57$ (d, $J=8.7 \mathrm{~Hz}$, 2H, ArH), 7.39 (d, $J=15.5 \mathrm{~Hz}, 1 \mathrm{H}, \mathrm{COCH}=\mathrm{CH}), 6.93$ (d, $J=8.4 \mathrm{~Hz}, 1 \mathrm{H}, \mathrm{ArH}), 6.70$ (d, $J=8.7 \mathrm{~Hz}, 2 \mathrm{H}, \mathrm{ArH}), 3.96$ $\left(\mathrm{d}, J=3.3 \mathrm{~Hz}, 6 \mathrm{H}, \mathrm{OCH}_{3}\right), 3.79(\mathrm{t}, J=6.9 \mathrm{~Hz}, 4 \mathrm{H}$, $\mathrm{CH}_{2} \mathrm{CH}_{2} \mathrm{Cl}$ ), 3.66 (t, $J=6.8 \mathrm{~Hz}, 4 \mathrm{H}, \mathrm{CH}_{2} \mathrm{CH}_{2} \mathrm{Cl}$ ); IR ( $\mathrm{KBr}$ ) v: 3448, 3073, 2927, 1649, 1561, 1513, 1455, 1350, 1258, 1171, 1022, 914, 816, $733 \mathrm{~cm}^{-1}$; HRMS (ESI) calcd for $\mathrm{C}_{21} \mathrm{H}_{23} \mathrm{Cl}_{2} \mathrm{NO}_{3} \mathrm{Na}[\mathrm{M}+\mathrm{Na}]^{+}$430.0947, found 430.0952.

(E)-4-[ $N, N$-二(2-氯乙基)-4-氨基-苯基]-1-(3',4'-二甲 苯基)-2-丙烯-1-酮(3k): 黄色固体, 收率 85\%. m.p. $106 \sim 108{ }^{\circ} \mathrm{C} ;{ }^{1} \mathrm{H}$ NMR (400 MHz, $\left.\mathrm{CDCl}_{3}\right) \delta: 7.81 \sim 7.71$ (m, 3H, ArH), 7.56 (d, $J=8.7 \mathrm{~Hz}, 2 \mathrm{H}, \mathrm{ArH}), 7.37$ (d, $J=$ $15.5 \mathrm{~Hz}, 1 \mathrm{H}, \mathrm{COCH}=\mathrm{CH}), 7.24(\mathrm{~d}, J=7.9 \mathrm{~Hz}, 1 \mathrm{H}$, $\mathrm{COCH}=\mathrm{CH}), 6.71(\mathrm{dd}, J=15.7,8.8 \mathrm{~Hz}, 2 \mathrm{H}, \mathrm{ArH}), 3.80$ (dd, $J=14.9,7.8 \mathrm{~Hz}, 4 \mathrm{H}, \mathrm{CH}_{2} \mathrm{CH}_{2} \mathrm{Cl}$ ), 3.66 (dd, $J=12.5$, $\left.5.8 \mathrm{~Hz}, 4 \mathrm{H}, \mathrm{CH}_{2} \mathrm{CH}_{2} \mathrm{Cl}\right), 2.34$ (d, $J=3.4 \mathrm{~Hz}, 6 \mathrm{H}, \mathrm{CH}_{3}$ ); IR (KBr) v: 3447, 2963, 2921, 1644, 1579, 1515, 1436, 1394, 1348, 1242, 1170, 1032, 980, 807, 738, $695 \mathrm{~cm}^{-1}$; HRMS
(ESI) calcd for $\mathrm{C}_{21} \mathrm{H}_{23} \mathrm{Cl}_{2} \mathrm{NONa}[\mathrm{M}+\mathrm{Na}]^{+}$389.1049, found 398.1059 .

(E)-4-[ $N, N$-二(2-氯乙基)-4-氨基-苯基]-1-(4'-甲氧基 苯基)-2-丙烯-1-酩(31): 黄色固体, 收率 87\%. m.p. 126 $128{ }^{\circ} \mathrm{C} ;{ }^{1} \mathrm{H}$ NMR (400 MHz, $\left.\mathrm{CDCl}_{3}\right) \delta: 8.08 \sim 7.98(\mathrm{~m}$, 2H, ArH), $7.76(\mathrm{~d}, J=15.5 \mathrm{~Hz}, 1 \mathrm{H}, \mathrm{COCH}=\mathrm{CH}), 7.57$ (d, $J=8.8 \mathrm{~Hz}, 2 \mathrm{H}, \mathrm{ArH}), 7.38$ (d, $J=15.5 \mathrm{~Hz}, 1 \mathrm{H}, \mathrm{COCH}=$ $\mathrm{CH}), 7.02 \sim 6.94(\mathrm{~m}, 2 \mathrm{H}, \mathrm{ArH}), 6.70(\mathrm{~d}, J=8.9 \mathrm{~Hz}, 2 \mathrm{H}$, ArH), $3.89\left(\mathrm{~s}, 3 \mathrm{H}, \mathrm{OCH}_{3}\right), 3.80(\mathrm{t}, J=7.0 \mathrm{~Hz}, 4 \mathrm{H}$, $\mathrm{CH}_{2} \mathrm{CH}_{2} \mathrm{Cl}$ ), 3.70 3.64 (m, 4H, $\mathrm{CH}_{2} \mathrm{CH}_{2} \mathrm{Cl}$ ); IR (KBr) v: 3445, 2960, 2927, 1645, 1585, 1516, 1345, 1256, 1171, 1019, 981, 820, $796 \mathrm{~cm}^{-1}$; HRMS (ESI) calcd for $\mathrm{C}_{20} \mathrm{H}_{21} \mathrm{Cl}_{2} \mathrm{NO}_{2} \mathrm{Na}[\mathrm{M}+\mathrm{Na}]^{+}$400.0842, found 400.0832.

(E)-4-[N, $N$-二(2-氯乙基)-4-氨基-苯基]-1-(4'-联苯 基)-2-丙烯-1-酮(3m): 浅黄色固体，收率 84\%. m.p. $128 \sim 130{ }^{\circ} \mathrm{C} ;{ }^{1} \mathrm{H}$ NMR (400 MHz, $\left.\mathrm{CDCl}_{3}\right) \delta: 8.09$ (d, $J=$ $8.3 \mathrm{~Hz}, 2 \mathrm{H}, \mathrm{ArH}), 7.80$ (d, $J=15.5 \mathrm{~Hz}, 1 \mathrm{H}, \mathrm{COCH}=\mathrm{CH})$, $7.72(\mathrm{~d}, J=8.3 \mathrm{~Hz}, 2 \mathrm{H}, \operatorname{ArH}), 7.65(\mathrm{~d}, J=7.3 \mathrm{~Hz}, 2 \mathrm{H}$, ArH), 7.59 (d, $J=8.8 \mathrm{~Hz}, 2 \mathrm{H}, \operatorname{ArH}), 7.48$ (t, $J=7.5 \mathrm{~Hz}$, $2 \mathrm{H}, \mathrm{ArH}, \mathrm{COCH}=\mathrm{CH}), 7.41(\mathrm{dd}, J=11.5,4.0 \mathrm{~Hz}, 2 \mathrm{H}$, ArH), $6.71(\mathrm{~d}, J=8.9 \mathrm{~Hz}, 2 \mathrm{H}, \operatorname{ArH}), 3.81(\mathrm{t}, J=6.9 \mathrm{~Hz}$, $4 \mathrm{H}, \mathrm{CH}_{2} \mathrm{CH}_{2} \mathrm{Cl}$ ), 3.67 (t, $J=6.9 \mathrm{~Hz}, 4 \mathrm{H}, \mathrm{CH}_{2} \mathrm{CH}_{2} \mathrm{Cl}$ ); IR (KBr) v: 2959, 2924, 2856, 1670, 1593, 1518, 1463, 1398, 1355, 1237, 1164, 812, 745, $707 \mathrm{~cm}^{-1}$; HRMS (ESI) calcd for $\mathrm{C}_{25} \mathrm{H}_{23} \mathrm{Cl}_{2} \mathrm{NONa}[\mathrm{M}+\mathrm{Na}]^{+}$446.1045, found 446.1049.

(E)-4-[ $N, N$-二(2-氯乙基)-4-氨基-苯基]-1-(2'-溴-4'氟苯基)-2-丙烯-1-醖(3n): 黄色固体, 收率 84\%. m.p. $73 \sim 75{ }^{\circ} \mathrm{C} ;{ }^{1} \mathrm{H}$ NMR (400 MHz, $\left.\mathrm{CDCl}_{3}\right) \delta: 7.48(\mathrm{~d}, J=8.7$ $\mathrm{Hz}, 2 \mathrm{H}, \mathrm{ArH}), 7.45 \sim 7.42(\mathrm{~m}, 1 \mathrm{H}, \mathrm{ArH}), 7.42 \sim 7.38(\mathrm{~m}$, $1 \mathrm{H}, \mathrm{ArH}), 7.35(\mathrm{~d}, J=16.3 \mathrm{~Hz}, 1 \mathrm{H}, \mathrm{COCH}=\mathrm{CH}), 7.12$ $(\mathrm{td}, J=8.2,2.2 \mathrm{~Hz}, 1 \mathrm{H}, \mathrm{ArH}), 6.91(\mathrm{~d}, J=15.9 \mathrm{~Hz}, 1 \mathrm{H}$, $\mathrm{COCH}=\mathrm{CH}), 6.71(\mathrm{dd}, J=23.2,8.8 \mathrm{~Hz}, 2 \mathrm{H}, \mathrm{ArH}), 3.81$ (dd, $\left.J=15.5,8.6 \mathrm{~Hz}, 4 \mathrm{H}, \mathrm{CH}_{2} \mathrm{CH}_{2} \mathrm{Cl}\right), 3.70 \sim 3.63(\mathrm{~m}, 4 \mathrm{H}$, $\mathrm{CH}_{2} \mathrm{CH}_{2} \mathrm{Cl}$ ); IR (KBr) v: 3032, 2923, 1642, 1589, 1516, 1479, 1351, 1250, 1178, 1093, 979, 863, $812 \mathrm{~cm}^{-1}$; HRMS (ESI) calcd for $\mathrm{C}_{19} \mathrm{H}_{17} \mathrm{BrCl}_{2} \mathrm{FNONa}[\mathrm{M}+\mathrm{Na}]^{+}$467.9724, found 467.9713 .

(E)-4-[ $N, N$-二(2-氯乙基)-4-氨基-苯基]-1-(2',5'-二甲 基苯基)-2-丙烯-1-酮(3o): 黄色固体，收率 78\%. m.p. $56 \sim 58{ }^{\circ} \mathrm{C} ;{ }^{1} \mathrm{H}$ NMR (400 MHz, $\left.\mathrm{CDCl}_{3}\right) \delta: 7.47$ (d, $J=8.4$ $\mathrm{Hz}, 2 \mathrm{H}, \mathrm{ArH}), 7.37$ (d, $J=15.9 \mathrm{~Hz}, 1 \mathrm{H}, \mathrm{COCH}=\mathrm{CH})$, 7.24 (s, 1H, ArH), 7.15 (q, $J=8.0 \mathrm{~Hz}, 2 \mathrm{H}, \mathrm{ArH}), 6.93$ (d, $J=15.9 \mathrm{~Hz}, 1 \mathrm{H}, \mathrm{COCH}=\mathrm{CH}), 6.67(\mathrm{~d}, J=8.5 \mathrm{~Hz}, 2 \mathrm{H}$, ArH), 3.78 (t, $J=6.8 \mathrm{~Hz}, 4 \mathrm{H}, \mathrm{CH}_{2} \mathrm{CH}_{2} \mathrm{Cl}$ ), 3.64 (t, $J=6.8$ $\mathrm{Hz}, 4 \mathrm{H}, \mathrm{CH}_{2} \mathrm{CH}_{2} \mathrm{Cl}$ ), 2.36 (d, J=4.9 Hz, 6H, $\mathrm{CH}_{3}$ ); IR 
(KBr) v: 3437, 2958, 2921, 1587, 1516, 1435, 1345, 1244 , 1172, 1025, 982, 813, $749 \mathrm{~cm}^{-1}$; HRMS (ESI) calcd for $\mathrm{C}_{21} \mathrm{H}_{23} \mathrm{Cl}_{2} \mathrm{NONa}[\mathrm{M}+\mathrm{Na}]^{+}$398.1049, found 398.1055.

(E)-4-[ $N, N$-二(2-氯乙基)-4-氨基-苯基]-1-苯基-2-丙 烯-1-酮(3p): 黄色固体, 收率 81\%. m.p. 90 92 ${ }^{\circ} \mathrm{C} ;{ }^{1} \mathrm{H}$ NMR (400 MHz, $\left.\mathrm{CDCl}_{3}\right) \delta: 8.04 \sim 7.97(\mathrm{~m}, 2 \mathrm{H}, \mathrm{ArH})$, $7.76(\mathrm{~d}, J=15.6 \mathrm{~Hz}, 1 \mathrm{H}, \mathrm{COCH}=\mathrm{CH}), 7.60 \sim 7.53(\mathrm{~m}$, $3 \mathrm{H}, \mathrm{ArH}), 7.49$ (t, $J=7.4 \mathrm{~Hz}, 2 \mathrm{H}, \mathrm{ArH}), 7.36$ (d, $J=15.6$ $\mathrm{Hz}, 1 \mathrm{H}, \mathrm{COCH}=\mathrm{CH}), 6.69(\mathrm{~d}, J=8.8 \mathrm{~Hz}, 2 \mathrm{H}, \mathrm{ArH}), 3.79$ (t, $J=6.9 \mathrm{~Hz}, 4 \mathrm{H}, \mathrm{CH}_{2} \mathrm{CH}_{2} \mathrm{Cl}$ ), 3.65 (t, $J=6.9 \mathrm{~Hz}, 4 \mathrm{H}$, $\mathrm{CH}_{2} \mathrm{CH}_{2} \mathrm{Cl}$ ); IR ( $\left.\mathrm{KBr}\right) v$ : 2962, 1648, 1584, 1514, 1455, 1395, 1351, 1214, 1018, 988, 814, 774, $735 \mathrm{~cm}^{-1}$; HRMS (ESI) calcd for $\mathrm{C}_{19} \mathrm{H}_{20} \mathrm{Cl}_{2} \mathrm{NO}[\mathrm{M}+\mathrm{H}]^{+} 348.0916$, found 348.0904 .

(E)-4-[ $N, N$-二(2-氯乙基)-4-氨基-苯基]-1-(2',4'-二烯 丙氧基苯基)-2-丙烯-1-酮(3q)：黄色固体，收率 86\%. m.p. 90 91 ${ }^{\circ} \mathrm{C}$; H NMR (400 MHz, $\left.\mathrm{CDCl}_{3}\right) \delta: 7.74$ (d, $J=8.6 \mathrm{~Hz}, 1 \mathrm{H}, \mathrm{ArH}), 7.64(\mathrm{~d}, J=15.7 \mathrm{~Hz}, 1 \mathrm{H}, \mathrm{COCH}=$ $\mathrm{CH}), 7.50$ (d, $J=8.7 \mathrm{~Hz}, 2 \mathrm{H}, \mathrm{ArH}), 7.43(\mathrm{~d}, J=15.7 \mathrm{~Hz}$, $1 \mathrm{H}, \mathrm{COCH}=\mathrm{CH}), 6.68(\mathrm{~d}, J=8.7 \mathrm{~Hz}, 2 \mathrm{H}, \mathrm{ArH}), 6.57(\mathrm{dd}$, $J=8.6,1.8 \mathrm{~Hz}, 1 \mathrm{H}, \mathrm{ArH}), 6.52(\mathrm{~d}, J=1.7 \mathrm{~Hz}, 1 \mathrm{H}, \mathrm{ArH})$, $6.11 \sim 6.00\left(\mathrm{~m}, 2 \mathrm{H}, \mathrm{CH}_{2} \mathrm{CH}=\mathrm{CH}_{2}\right), 5.45(\mathrm{dd}, J=17.2$, $\left.10.7 \mathrm{~Hz}, 2 \mathrm{H}, \mathrm{CH}_{2} \mathrm{CH}=\mathrm{CH}_{2}\right), 5.30(\mathrm{dd}, J=16.7,10.6 \mathrm{~Hz}$, $\left.2 \mathrm{H}, \mathrm{CH}_{2} \mathrm{CH}=\mathrm{CH}_{2}\right), 4.61\left(\mathrm{~d}, J=4.8 \mathrm{~Hz}, 2 \mathrm{H}, \mathrm{OCH}_{2} \mathrm{CH}=\right.$ $\left.\mathrm{CH}_{2}\right), 4.59\left(\mathrm{~d}, J=5.2 \mathrm{~Hz}, 2 \mathrm{H}, \mathrm{OCH}_{2} \mathrm{CH}=\mathrm{CH}_{2}\right), 3.78(\mathrm{t}$, $\left.J=6.9 \mathrm{~Hz}, 4 \mathrm{H}, \mathrm{CH}_{2} \mathrm{CH}_{2} \mathrm{Cl}\right), 3.66(\mathrm{t}, J=6.9 \mathrm{~Hz}, 4 \mathrm{H}$, $\mathrm{CH}_{2} \mathrm{CH}_{2} \mathrm{Cl}$ ); IR (KBr) v: 3082, 2961, 1645, 1598, 1514, 1425, 1393, 1287, 1178, 1117, 1013, 949, 805, $738 \mathrm{~cm}^{-1}$; HRMS (ESI) calcd for $\mathrm{C}_{25} \mathrm{H}_{28} \mathrm{Cl}_{2} \mathrm{NO}_{3}[\mathrm{M}+\mathrm{H}]^{+}$460.1441, found 460.1458 .

(E)-4-[ $N, N$-二 (2- 氯乙基)-4-氨基-3- 苯基 ]-1- $\{3$ '$[N, N$-二(2-羟乙基) $]$-苯基 $\}$-2-丙烯-1-酮(3r): 黄色液体, 收率 $88 \% .{ }^{1} \mathrm{H}$ NMR (400 MHz, $\left.\mathrm{CDCl}_{3}\right) \delta: 7.66(\mathrm{~d}, J=15.5$ $\mathrm{Hz}, 1 \mathrm{H}, \mathrm{COCH}=\mathrm{CH}), 7.50(\mathrm{~d}, J=8.7 \mathrm{~Hz}, 2 \mathrm{H}, \mathrm{ArH}), 7.28$ (s, 1H, ArH), $7.25 \sim 7.19$ (m, 3H, ArH), 6.84 (d, $J=7.6$ $\mathrm{Hz}, 1 \mathrm{H}, \mathrm{COCH}=\mathrm{CH}), 6.65(\mathrm{~d}, J=8.7 \mathrm{~Hz}, 2 \mathrm{H}, \mathrm{ArH}), 4.73$ (s, $2 \mathrm{H}, \mathrm{OH}), 3.79$ (d, J=4.8 Hz, $\left.4 \mathrm{H}, \mathrm{CH}_{2} \mathrm{CH}_{2} \mathrm{OH}\right), 3.78 \sim$ $3.71\left(\mathrm{~m}, 4 \mathrm{H}, \mathrm{CH}_{2} \mathrm{CH}_{2} \mathrm{Cl}\right), 3.63$ (t, $J=6.8 \mathrm{~Hz}, 4 \mathrm{H}$, $\mathrm{CH}_{2} \mathrm{CH}_{2} \mathrm{Cl}$ ), 3.56 (d, $J=4.5 \mathrm{~Hz}, 4 \mathrm{H}, \mathrm{CH}_{2} \mathrm{CH}_{2} \mathrm{OH}$ ); IR (KBr) v: 3374, 2928, 2878, 1672, 1568, 1517, 1442, 1353, 1268, 1177, 1066, 1005, 817, $742 \mathrm{~cm}^{-1}$; HRMS (ESI) calcd for $\mathrm{C}_{23} \mathrm{H}_{28} \mathrm{Cl}_{2} \mathrm{~N}_{2} \mathrm{O}_{3} \mathrm{Na}[\mathrm{M}+\mathrm{Na}]^{+} 473.1369$, found 473.1365 .

\section{2 结果与讨论}

\section{1 合成}

以苯胺为原料, 经羟乙基化、改变的 Vilsmeier 反 应、羟醛缩合得到目标化合物 3a $\sim 3 \mathbf{r}$. 中间体 $N, N$ 二 (2氯乙基)-4-氨基-苯甲醛(2)是合成目标产物的关键步骤, 对反应条件优化表明 $\mathrm{DMF}$ 与 $\mathrm{POCl}_{3}$ 的反应时间和反应 投料比对收率有重要影响. 当 $\mathrm{DMF}$ 与 $\mathrm{POCl}_{3}$ 在 $0{ }^{\circ} \mathrm{C}$ 反 应约 $45 \mathrm{~min}$, 反应物料比分别是: $n(N, N$-二羟乙基苯 胺) $: n\left(\mathrm{POCl}_{3}\right)=1: 3.3, m$ (总 DMF) $: m(N, N-$ 二羟乙基 苯胺 $)=4: 1, m_{1}\left(\mathrm{POCl}_{3}\right.$ 加入前烧瓶中的 $\left.\mathrm{DMF}\right): m_{2}$ (溶 解 $N, N$-二羟乙基苯胺的 $\mathrm{DMF}$ ) $=3: 1$ 时, 对-[二(2-氯乙 基)]胺基苯甲醛的产率为 $95 \%$. 整个合成反应操作简单， 产物容易分离, 总产率较高.

\section{2 晶体结构}

单晶 $\mathrm{X}$ 射线衍射测试表明化合物 $\mathbf{3 e}, \mathbf{3 I}$ 的晶体结构 都属三斜晶系(Triclinic), $P-1$ 空间群. 化合物 $3 \mathbf{e}$ 的分子 式为 $\mathrm{C}_{19} \mathrm{H}_{18} \mathrm{BrCl}_{2} \mathrm{NO}$, 晶胞参数为 $a=8.129(2) \AA, b=$ 9.387(3) $\AA, c=12.911(4) \AA, \alpha=75.548(5)^{\circ}, \beta=$ $79.105(5)^{\circ}, \gamma=79.013(5)^{\circ}, \quad V=926.1(5) \AA^{3}, D_{\mathrm{c}}=1.532$ $\mathrm{Mg} / \mathrm{m}^{3}, \mu=2.513 \mathrm{~mm}^{-1}, \mathrm{Z}=2, F(000)=432$; 化合物 $3 \mathrm{I}$ 的分子式为 $\mathrm{C}_{20} \mathrm{H}_{21} \mathrm{Cl}_{2} \mathrm{NO}_{2}$, 晶胞参数分别为 $a=8.361(2)$ $\AA, b=9.154(3) \AA, c=12.288(3) \AA, \alpha=81.657(5)^{\circ}, \beta=$ $84.525(4)^{\circ}, \gamma=83.138(5)^{\circ}, \quad V=920.9(4) \AA^{3}, D_{\mathrm{c}}=1.364$ $\mathrm{Mg} / \mathrm{m}^{3}, \mu=0.366 \mathrm{~mm}^{-1}, \mathrm{Z}=2, F(000)=396$. 化合物 $3 \mathbf{e}$ 和 31 的晶体数据已存于剑桥晶体数据库, 其 $\mathrm{CCDC}$ 号分 别为 886492 和 886494 .

晶体结构分析表明：化合物 $3 \mathrm{e}$ 和 31 分子结构中, 由 于两苯环上取代基的空间效应，使分子中两苯环都不在 同一平面上, 其二面角分别为 $17.9^{\circ}$ 和 $19.42^{\circ}$. 化合物 3e, 31 的晶体结构见图 1. 我们对化合物 $3 \mathrm{e}$ 和 31 的晶体 数据进行了比较, 化合物 $3 \mathrm{e}, 3 \mathrm{I}$ 分子中都存在微弱的氢 键，化合物 3e 通过 $\mathrm{C}(9)-\mathrm{H}(9) \cdots \mathrm{O}(1)$ 形成分子间氢键, 化合物 $3 \mathrm{I}$ 通过 $\mathrm{C}(11)-\mathrm{H}(11) \cdots \mathrm{O}(1)$ 形成分子间氢键, 以 交错形式形成紧密排列. 化合物 3e, 31 的晶体结构见图 1 , 晶胞堆积图、氢键数据、部分键长键角数据见辅助材 料.

\section{3 抗肿瘤活性测试}

以市售抗肿瘤药物阿霉素(AMD)和顺铂(DDP)为阳 性对照, 采用 MTT [3-(4,5-dimethylthiazol-2-yl)-5iphenyltetrazolium bromide]比色实验, 对合成的含氮 芥基的查尔酮类衍生物对 K562(人髓系白血病癌细 胞)和 HepG2(人源肝癌细胞)分别进行了体外抗肿瘤活 

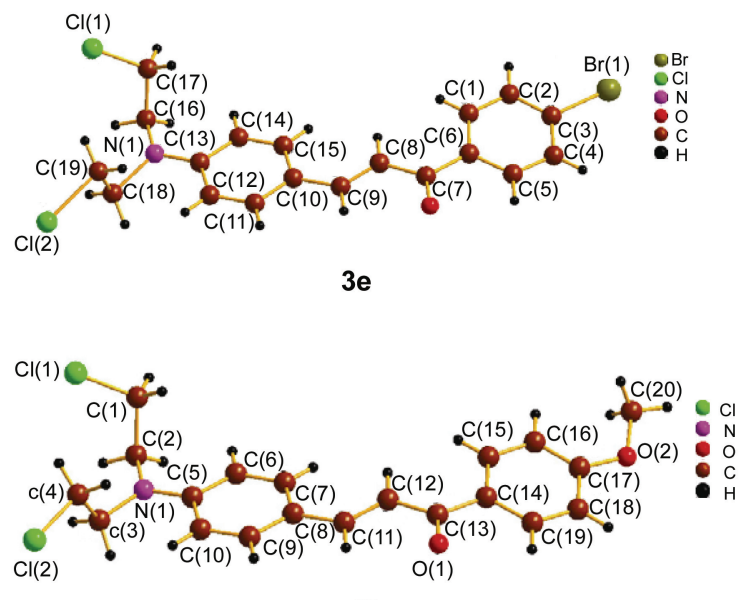

3I

图 1 化合物 $3 \mathrm{e}$ 和 31 的晶体结构

Figure 1 Crystal structures of compounds $\mathbf{3 e}$ and $\mathbf{3 1}$

性的初步篎选. 取对数生长期的肿瘤细胞, 细胞悬浮液 为含 $5 \%$ 灭活牛血清、双抗(青霉素 $100 \mathrm{U} / \mathrm{mL}$, 链霨素 $100 \mu \mathrm{g} / \mathrm{mL}$ ) 的 RPMI 1640 培养基, 调整细胞浓度为 $1 \times 10^{5}$ 个 $/ \mathrm{mL}$ 加于 96 孔板中, $100 \mu \mathrm{L} /$ 孔. 96 孔培养板 分组设置如图 2 所示: $2 \sim 9$ 列为样品组、第 10 列为 细胞对照组(不加样品)、第 11 列为空白组(只含培养 基), $\mathrm{A}$ 行 2 9 孔为药品对照组(只含样品), 每组设 6 个复孔. 于饱和湿度, $5 \% \mathrm{CO}_{2}, 37{ }^{\circ} \mathrm{C}$ 条件下培养 $24 \mathrm{~h}$, 将用培养液连续二倍稀释的样品分别加入样品孔中, 每孔 $100 / \mu \mathrm{L}$, 使得样品组最终浓度为 $256,128,64,32$, $16,8,4,2 \mu \mathrm{g} / \mathrm{L}$, 其它各设置孔加 $100 \mu \mathrm{L}$ 培养基. 孵育 72 $\mathrm{h}$, 每孔加入 MTT 溶液 $10 \mu \mathrm{L}(5 \mathrm{mg} / \mathrm{mL}), 37{ }^{\circ} \mathrm{C}$ 作用 $4 \mathrm{~h}$ 后, 小心洗去孔内液体, 加入 DMSO $150 \mu \mathrm{L}$ 孔, 室温 振摇 $10 \mathrm{~min}$, 用酶联免疫检测仪测 $490 \mathrm{~nm}$ OD 值. 根据 细胞增殖抑制率公式计算: 抑制率 $=(O D$ 样品 $\mathrm{OD}$ 样品对照组 $) /(\mathrm{OD}$ 细胞对照组 $-O D$ 空白组 $) \times 100 \% . \mathrm{IC}_{50}$ 值用统 计软件 Spass 13.0 进行计算, 实验结果见表 1 .

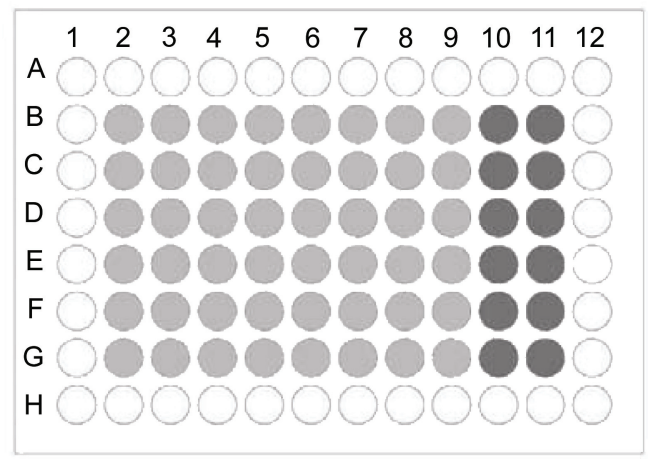

图 296 孔板 $(2 \sim 11$ 列实验组为阴影 $)$

Figuer 2 A 96-well plate (columns $2 \sim 11$ in the shaded wells) 测定结果表明, 此类化合物对两种细胞呈现出选择
性抑制效果，其中化合物 $\mathbf{3 j}$ 和 $\mathbf{3 1}$ 的抑制活性最强，对所 选两种细胞都显示出较强的抑制作用，对 K562 的抑制 活性与阿霉素相近，对 HepG2 的抑制活性分别为顺铂 的 4 倍和 2 倍. 化合物 $3 \mathbf{b}, \mathbf{3 c}, \mathbf{3 e}, \mathbf{3 i}, \mathbf{3 m}, \mathbf{3 o}$ 和 $3 \mathbf{r}$ 只对 K562 细胞有选择性抑制作用，化合物 3k 只显示出对 HepG2 有抑制作用, 而化合物 3d 则对两种细胞都无抑 制效果. 由表 1 可看出, 除化合物 3d 和 3k 外, 其余化 合物对 K562 细胞显示出不同程度高于顺铂的抑制活性, 而低于阿霉素的抑制作用. 所合成的目标化合物对 HepG2 细胞的抑制活性则不同程度低于顺铂和阿霉素. 此类化合物中苯环上不同取代基可能影响到对肿瘤细 胞的选择性抑制，可以看出，苯环中含有甲基 $\mathbf{3 j}, \mathbf{3 1}$ 时, 化合物对两种细胞都有很好的抑制作用, 且随甲氧基数 目增多, 活性更显著, 实验结果与文献相关报道一致 [22]. 苯环上含有着基 3d 时, 则对两种癌细胞都无抑制 作用. 苯环上含有单卤原子 $\mathbf{3 b}, \mathbf{3 c}, \mathbf{3 e}, \mathbf{3 f}$ 时, 目标化合 物对 K562 细胞呈现出优于 HepG2 的抑制效果, 而苯环 上含有多卤原子 $3 n$ 时，目标化合物对 HepG2 细胞的抑 制活性明显强于 K562. 对比苯环上不同取代基电子效 应对目标化合物抗肿瘤活性的影响时，并没有发现明显 的规律. 对此类化合物的合成和抗肿瘤活性的深入研究 正在进行中.

表 1 目标化合物对 $\mathrm{K} 562$ 和 $\mathrm{HepG} 2$ 的 $\mathrm{IC}_{50}$ 值 $(\mu \mathrm{mol} / \mathrm{L})^{a}$

Table $1 \mathrm{IC}_{50}(\mu \mathrm{mol} / \mathrm{L})$ values of target compounds for $\mathrm{K} 562$ and HepG2

\begin{tabular}{ccc||ccc}
\hline Compd. & K562 & HepG2 & Compd. & K562 & HepG2 \\
\hline $\mathbf{3 a}$ & 51.35 & 94.38 & $\mathbf{3 k}$ & $\mathrm{NA}^{b}$ & 50.17 \\
$\mathbf{3 b}$ & 115.73 & $\mathrm{NA}$ & $\mathbf{3 l}$ & 18.66 & 21.39 \\
$\mathbf{3 c}$ & 100.58 & $\mathrm{NA}$ & $\mathbf{3 m}$ & 63.47 & $\mathrm{NA}$ \\
$\mathbf{3 d}$ & $\mathrm{NA}$ & $\mathrm{NA}$ & $\mathbf{3 n}$ & 81.41 & 33.21 \\
$\mathbf{3 e}$ & 49.75 & $\mathrm{NA}$ & $\mathbf{3 o}$ & 179.93 & $\mathrm{NA}$ \\
$\mathbf{3 f}$ & 62.56 & 123.31 & $\mathbf{3 p}$ & 63.11 & 142.68 \\
$\mathbf{3 g}$ & 136.38 & 34.64 & $\mathbf{3 q}$ & 101.27 & 68.94 \\
$\mathbf{3 h}$ & 153.81 & 56.05 & $\mathbf{3 r}$ & 42.19 & $\mathrm{NA}$ \\
$\mathbf{3 i}$ & 122.94 & $\mathrm{NA}$ & $\mathrm{DDP}$ & $\mathrm{NA}$ & 11.31 \\
$\mathbf{3 j}$ & 20.01 & 43.64 & $\mathrm{AMD}$ & 14.88 & 4.19 \\
\hline
\end{tabular}

${ }^{a} \mathrm{IC}_{50}$ values mean of three experiments in replicate; ${ }^{b}$ means inactive $\mathrm{IC}_{50}$ values $>200 \mu \mathrm{mol} \cdot \mathrm{L}^{-1}$.

辅助材料(Supporting Information) 化合物 $3 \mathrm{e}$ 和 31 的 晶胞堆积图、氢键及部分键长键角数据. 这些材料可以 免费从本刊网站(http://sioc-journal.cn/)上下载.

\section{References}

[1] Haraguchi, H.; Ishikawa, H.; Mizutani, K.; Tamura, Y.; Kinoshita, T. Bioorg. Med. Chem. 1998, 6, 339.

[2] Kumar, S. K.; Hager, E.; Pettit, C.; Gurulingappa, H.; Davidson, N. E.; Khan, S. R. J. Med. Chem. 2003, 46, 2813.

[3] Herencia, F.; Ferrandiz, M. L.; Ubeda, A.; Dominguez, J. N.; Charris, J. E.; Lobo, G. M.; Alcaraz, M. J. Bioorg. Med. Chem. Lett. 
1998, 8,1169

[4] Ni, L.-M.; Meng, C.-Q.; Sikorski, J. A. Expert Opin. Ther. Pat. 2004, 14, 1669.

[5] Trivedi, J. C.; Bariwal, J. B.; Upadhyay, K. D.; Naliapara, Y. T.; Joshi, S. K.; Pannecouque, C. C.; Clercq, E. D.; Shaha, A. K. Tetrahedron Lett. 2007, 48, 8472.

[6] Nowakowska, Z.; Kedzia, B.; Schroeder, G. Eur. J. Med. Chem. 2008, 43, 707.

[7] Lopez, S. N.; Castelli, M. V.; Zacchino, S. A.; Domınguez, J. N.; Lobo, G.; Charris-Charris, C.; Cortes, J. C. G.; Ribas, J. C.; Devia, C.; Rodriguez, A. M.; Enriz, R. D. Bioorg. Med. Chem. 2001, 9, 1999.

[8] Murakami, S.; Muramatsu, M.; Aihara, H. Biochem. Pharmacol. 1991, 42, 1447.

[9] Hseu, Y. C.; Lee, M. S.; Wu, C.-R.; Cho, H.-J.; Lin, K.-Y.; Lai, G.-H.; Wang, S.-Y.; Kuo, Y.-H.; Kumar, K. J. S.; Yang, H. L. J. Agric. Food Chem. 2012, 60, 2385.

[10] Kumar, S. K.; Hager, E.; Pettit, C.; Gurulingappa, H.; Davidson, N. E.; Khan, S. R. J. Med. Chem. 2003, 46, 2813.

[11] Lorenzo, P.; Alvarez, R.; Ortiz, M. A.; Alvarez, S.; Piedrafita, F. J.; Lera, A. R. J. Med. Chem. 2008, 51, 5431.

[12] Tatsuzaki, J.; Bastow, K. F.; Nakagawa-Goto, K.; Nakamura, S.; Itokawa, H.; Lee, K. H. J. Nat. Prod. 2006, 69, 1445.

[13] Rajski, S. R.; Williams, R. M. Chem. Rev. 1998, 98, 2723.
[14] Rink, S. M.; Hopkins, P. B. Bioorg. Med. Chem. Lett. 1995, 5, 2845.

[15] Catovsky, D.; Else, M.; Richards, S. Clin. Lymphoma, Myeloma Leuk. 2011, 11, S2.

[16] Kapoor, P.; Rajkumar, S. V.; Dispenzieri, A.; Gertz, M. A.; Lacy, M. Q.; Dingli, D.; Mikhael, J. R.; Roy, V.; Kyle, R. A.; Greipp, P. R.; Kumar, S.; Mandrekar, S. J. Leukemia 2011, 25, 689.

[17] Rappeneau, S.; Squiban, A. B.; Boucher, F. B.; Aubery, M.; Gendron, M. C.; Marano, F. Toxicol. In Vitro 1999, 13, 765.

[18] Ren, J.; Xu, H.-J.; Cheng, H.; Xin, W.-Q.; Chen, X.; Hu, K. Eur. J. Med. Chem. 2012, 54, 175.

[19] Zhuang, Y.-Y.; Zhou, C.-H.; Wang, Y.-F.; Li, D.-H. Chin. Pharm. $J$. 2008, 17, 1281 (in Chinese).

(庄雅云, 周成合, 王渝芳, 李东红, 中国药学杂志, 2008, 17, 1281.)

[20] Xu, F.-H.; Gan, Y.; Zhang, Y.-H.; Luo, W.; Ma, H.-X.; Wang, C.-J.; Zhao, J. Chin. J. Org. Chem. 2011, 31, 1122 (in Chinese). (许风华, 甘荣, 张亚宏, 罗稳, 马红霞, 王超杰, 赵瑾, 有机化 学, 2011, 31, 1122.)

[21] Tang, Y.-D.; Zhang, J.-Q.; Zhang, S.-L.; Geng, R.-X.; Zhou, C.-H. Chin. J. Chem. 2012, 30, 1831.

[22] Srinivasan, B.; Johnson, T. E.; Lad, R.; Xing, C.-G. J. Med. Chem. 2009, 52,7228 . 